\title{
Small Pre-Quasi Banach Operator Ideals of Type Orlicz-Cesáro Mean Sequence Spaces
}

\author{
Awad A. Bakery $\mathbb{D}^{1,2}$ and Mustafa M. Mohammed $\mathbb{D}^{1,3}$ \\ ${ }^{1}$ Department of Mathematics, Faculty of Science and Arts, University of Jeddah(UJ), P.O. Box 355, Code 21921 Khulais, Saudi Arabia \\ ${ }^{2}$ Department of Mathematics, Faculty of Science, Ain Shams University, P.O. Box 1156, Abbassia, Cairo 11566, Egypt \\ ${ }^{3}$ Department of Statistics, Faculty of Science, Sudan University of Science \& Technology, Khartoum, Sudan
}

Correspondence should be addressed to Mustafa M. Mohammed; mustasta@gmail.com

Received 11 February 2019; Accepted 15 April 2019; Published 2 May 2019

Guest Editor: Tuncer Acar

Copyright (C) 2019 Awad A. Bakery and Mustafa M. Mohammed. This is an open access article distributed under the Creative Commons Attribution License, which permits unrestricted use, distribution, and reproduction in any medium, provided the original work is properly cited.

In this paper, we give the sufficient conditions on Orlicz-Cesáro mean sequence spaces $c e s_{\varphi}$, where $\varphi$ is an Orlicz function such that the class $S_{c e s_{\varphi}}$ of all bounded linear operators between arbitrary Banach spaces with its sequence of $s$-numbers which belong to $\operatorname{ces}_{\varphi}$ forms an operator ideal. The completeness and denseness of its ideal components are specified and $S_{c e s_{\varphi}}$ constructs a pre-quasi Banach operator ideal. Some inclusion relations between the pre-quasi operator ideals and the inclusion relations for their duals are explained. Moreover, we have presented the sufficient conditions on $\operatorname{ces}_{\varphi}$ such that the pre-quasi Banach operator ideal generated by approximation number is small. The above results coincide with that known for ces $p(1<p<\infty)$.

\section{Introduction}

Throughout the paper, by $w$, we mean the space of all real sequences, $\mathbb{R}$ the real numbers, and $\mathbb{N}=\{0,1,2, \ldots\}$ and $\mathfrak{L}(X, Y)$ the space of all bounded linear operators from a normed space $X$ into a normed space $Y$. The operator ideals theory takes an importance in functional analysis, since it has numerous applications in fixed point theorem, geometry of Banach spaces, spectral theory, eigenvalue distributions theorem, etc. Some of the operator ideals in the class of normed spaces or Banach spaces in functional analysis are characterized by various scalar sequence spaces. For example the ideal of compact operators is defined by kolmogorov numbers and the space $c_{0}$ of convergent to zero sequences. Pietsch [1] inspected the operator ideals framed by the approximation numbers and the classical sequence space $\ell^{p}(0<p<\infty)$. He proved that the ideals of Hilbert Schmidt operators and nuclear operators between Hilbert spaces are defined by $\ell^{2}$ and $\ell^{1}$, respectively, and the sequence of approximation numbers. In [2], Faried and Bakery examined the operator ideals developed by generalized Cesáro, Orlicz sequence spaces $\ell_{M}$, and the approximation numbers. In [3],
Faried and Bakery studied the operator ideals constructed by $s-$ numbers, generalized Cesáro and Orlicz sequence spaces $\ell_{M}$ and show that the operator ideal formed by the previous sequence spaces and approximation numbers is small under certain conditions. Also summation process and sequences spaces applications are closely related to Korovkin type approximation theorems and linear positive operators studied by Costarelli and Vinti [4] and Altomare [5]. The idea of this paper is to examine a generalized class $S_{c e s_{\varphi}}$ by using Orlicz-Cesáro mean sequence spaces $\operatorname{ces}_{\varphi}$ and the sequence of $s$-numbers, for which $S_{c e s_{\varphi}}$ constructs an operator ideal. The components of $S_{c e s_{\varphi}}$ as a pre-quasi Banach operator ideal containing finite dimensional operators as a dense subset and its completeness are proved. The inclusion relations between the pre-quasi operator ideals and the inclusion relations for their duals are determined. Finally, we show that the prequasi Banach operator ideal formed by the approximation numbers and ces $_{\varphi}$ is small under certain conditions. These results coincide with that known for ces $s_{p},(1<p<\infty)$ in [3]. Furthermore we give some examples which support our main results. 


\section{Definitions and Preliminaries}

Definition 1 (see [6]). The sequence $\left(s_{n}(T)\right)_{n=0}^{\infty}$, for all $T \in$ $\mathfrak{L}(X, Y)$ is named an $s$-function and the number $s_{n}(T)$ is called the $n^{\text {th }} s$ - number of $T$ if the following are satisfied:

(a) monotonicity: $\|T\|=s_{0}(T) \geq s_{1}(T) \geq s_{2}(T) \geq \cdots \geq$ 0 for all $T \in \mathfrak{Q}(X, Y)$;

(b) additivity: $s_{m+n-1}\left(T_{1}+T_{2}\right) \leq s_{m}\left(T_{1}\right)+s_{n}\left(T_{2}\right)$ for all $T_{1}, T_{2} \in \mathfrak{Q}(X, Y), m, n \in \mathbb{N}$

(c) property of ideal: $s_{n}(R T P) \leq\|R\| s_{n}(T)\|P\|$ for all $P \in \mathfrak{L}\left(X_{0}, X\right), T \in \mathfrak{Q}(X, Y)$, and $R \in \mathfrak{Q}\left(Y, Y_{0}\right)$, where $X_{0}$ and $Y_{0}$ are normed spaces;

(d) $s_{n}(\beta T)=|\beta| s_{n}(T)$ for every $T \in \mathfrak{L}(X, Y), \beta \in \mathbb{R}$;

(e) rank property: if $\operatorname{rank}(T) \leq n$ then $s_{n}(T)=0$ for every $T \in \mathfrak{L}(X, Y)$

(f) property of norming:

$$
s_{i}\left(I_{j}\right)= \begin{cases}1, & \text { if } i<j \\ 0, & \text { if } i \geq j\end{cases}
$$

where $I_{j}$ is the identity operator on $\mathbb{R}^{j}$.

There are a few instances of $s$-numbers; we notice the accompanying conditions:

(1) The n-th approximation number, denoted by $\alpha_{n}(T)$, is defined by $\alpha_{n}(T)=\inf \{\|T-B\|: B \in$ $\mathfrak{L}(X, Y)$ and $\operatorname{rank}(B) \leq n\}$.

(2) The $n$-th Hilbert number, denoted by $h_{n}(T)$, is defined by

$$
\begin{aligned}
& h_{n}(T)=\sup \left\{\alpha_{n}(A T B):\left\|A: Y \longrightarrow \ell_{2}\right\|\right. \\
& \left.\quad \leq 1 \text { and }\left\|B: \ell_{2} \longrightarrow X\right\| \leq 1\right\} .
\end{aligned}
$$

(3) The n-th Weyl number, denoted by $x_{n}(T)$, is defined by

$$
x_{n}(T)=\inf \left\{\alpha_{n}(T B):\left\|B: \ell_{2} \longrightarrow X\right\| \leq 1\right\} .
$$

(4) The n-th Kolmogorov number, denoted by $d_{n}(T)$, is defined by

$$
d_{n}(T)=\inf _{\operatorname{dim} Y \leq n} \sup _{\|x\| \leq 1} \inf _{y \in Y}\|T x-y\| .
$$

(5) The n-th Gel'fand number, denoted by $c_{n}(T)$, is defined by $c_{n}(T)=\alpha_{n}\left(J_{Y} T\right)$, where $J_{Y}$ is a metric injection from the space $Y$ to a higher space $l_{\infty}(\Psi)$ for an adequate index set $\Psi$. This number is independent of the choice of the higher space $l_{\infty}(\Psi)$.

(6) The $n$-th Chang number, denoted by $y_{n}(T)$, is defined by

$$
y_{n}(T)=\inf \left\{\alpha_{n}(A T):\left\|A: Y \longrightarrow \ell_{2}\right\| \leq 1\right\} .
$$

Remark 2 (see [6]). Among all the s-number sequences characterized above, it is easy to check that the approximation number, $\alpha_{n}(T)$, is the largest and the Hilbert number, $h_{n}(T)$, is the smallest $s$-number sequence, i.e., $h_{n}(T) \leq s_{n}(T) \leq \alpha_{n}(T)$ for any bounded linear operator $T$. If $T$ is defined on a Hilbert space and compact, then all the $s$-numbers correspond with the eigenvalues of $|T|$, where $|T|=\left(T^{*} T\right)^{1 / 2}$.

Theorem 3 ([6], p.115). Let $T \in \mathfrak{Q}(X, Y)$. Then

$$
\begin{aligned}
& h_{n}(T) \leq x_{n}(T) \leq c_{n}(T) \leq \alpha_{n}(T), \\
& h_{n}(T) \leq y_{n}(T) \leq d_{n}(T) \leq \alpha_{n}(T) .
\end{aligned}
$$

Theorem 4 ([6], p.90). An s-number sequence is called injective if, for any metric injection $K \in \mathfrak{Q}\left(Y, Y_{0}\right), s_{n}(T)=s_{n}(K T)$ for all $T \in \mathfrak{Q}(X, Y)$.

Theorem 5 ([6], p.95). An s-number sequence is called surjective if, for any metric surjection $P \in \mathfrak{Q}\left(X_{0}, X\right), s_{n}(T)=s_{n}(T P)$ for all $T \in \mathfrak{Q}(X, Y)$.

Theorem 6 ([6], pp.90-94). The Weyl and Gel'fand numbers are injective.

Theorem 7 ([6], pp.95). The Chang and Kolmogorov numbers are surjective.

Definition 8. A finite rank operator is a bounded linear operator whose dimension of the range space is finite.

Definition 9 ((dual $s$-numbers) [7]). For each $s$-number sequence $s=\left(s_{n}\right)$, a dual $s$-number function $s^{d}=\left(s_{n}^{d}\right)$ is defined by

$$
s_{n}^{d}(T)=s_{n}\left(T^{\prime}\right) \quad \text { for all } T \in \mathfrak{Q}(X, Y),
$$

where $T^{\prime}$ is the dual of $T$.

Definition 10 ([8], p.152)). An $s$-number sequence is called symmetric if $s_{n}(T) \geq s_{n}\left(T^{\prime}\right)$ for all $T \in \mathfrak{Q}(X, Y)$. If $s_{n}(T)=$ $s_{n}\left(T^{\prime}\right)$, then the $s$-number sequence is said to be completely symmetric.

Presently we express some known results of dual of an $s$ number sequence.

Theorem 11 ([8], p.152). The approximation numbers are symmetric, i.e., $\alpha_{n}\left(T^{\prime}\right) \leq \alpha_{n}(T)$ for $T \in \mathfrak{L}(X, Y)$.

Remark 12 (see [9]). $\alpha_{n}(T)=\alpha_{n}\left(T^{\prime}\right)$ for every compact operator $T$.

Theorem 13 ([8], p.153). Let $T \in \mathfrak{L}(X, Y)$. Then

$$
\begin{gathered}
c_{n}\left(T^{\prime}\right) \leq d_{n}(T), \\
c_{n}(T)=d_{n}\left(T^{\prime}\right) .
\end{gathered}
$$

In addition, if $T$ is a compact operator then $d_{n}(T)=c_{n}\left(T^{\prime}\right)$. 
Theorem 14 ([6], p.96). Let $T \in \mathfrak{Q}(X, Y)$. Then

$$
\begin{gathered}
y_{n}\left(T^{\prime}\right) \leq x_{n}(T), \\
x_{n}(T)=y_{n}\left(T^{\prime}\right),
\end{gathered}
$$

i.e., Chang numbers and Weyl numbers are dual to each other.

Theorem 15 ([8], p.153). The Hilbert numbers are completely symmetric, i.e., $h_{n}\left(T^{\prime}\right)=h_{n}(T)$ for all $T \in \mathfrak{Q}(X, Y)$.

Definition 16 (see $[10,11])$. The operator ideal $\mathbb{U}:=$ $\{\mathbb{U}(X, Y) ; X$ and $Y$ are Banach Spaces $\}$ is a subclass of linear bounded operators such that its components $\mathbb{U}(X, Y)$ which are subsets of $\mathfrak{Q}(X, Y)$ fulfill the accompanying conditions:

(i) $I_{A} \in \mathbb{U}$ where $A$ indicates one dimensional Banach space, where $\mathbb{U} \subset \mathbf{L}$.

(ii) For $T_{1}, T_{2} \in \mathbb{U}(X, Y)$, then $\beta_{1} T_{1}+\beta_{2} T_{2} \in \mathbb{U}(X, Y)$ for any scalars $\beta_{1}, \beta_{2}$.

(iii) If $T \in \mathfrak{R}\left(X_{0}, X\right), R \in \mathbb{U}(X, Y)$, and $P \in \mathfrak{R}\left(Y, Y_{0}\right)$, then $P R T \in \mathbb{U}\left(X_{0}, Y_{0}\right)$.

Definition 17 (see $[12,13]$ ). An Orlicz function is a function $\varphi:[0, \infty) \longrightarrow[0, \infty)$, which is nondecreasing, convex, and continuous with $\varphi(0)=0$ and $\varphi(x)>0$ for $x>0$ and $\lim _{x \rightarrow \infty} \varphi(x)=\infty$.

Definition 18. An Orlicz function $\varphi$ is said to satisfy $\Delta_{2}$ condition for every values of $x \geq 0$, if there is $a>0$, such that $\varphi(2 x) \leq a \varphi(x)$. The $\Delta_{2}$-condition is corresponding to $\varphi(m x) \leq a m \varphi(x)$ for every values of $m>1$ and $x$.

Lindenstrauss and Tzafriri [14] utilized the idea of an Olicz function to define Orlicz sequence space:

$$
\begin{aligned}
& \ell_{\varphi}=\{x \in \omega: \rho(\lambda x)<\infty \text { for some } \lambda>0\} \\
& \text { where } \rho(x)=\sum_{k=0}^{\infty} \varphi\left(\left|x_{k}\right|\right),
\end{aligned}
$$

$\left(\ell_{\varphi},\|\|.\right)$ is a Banach space with the Luxemburg norm:

$$
\|x\|_{\ell_{\varphi}}=\inf \left\{\lambda>0: \rho\left(\lambda^{-1} x\right) \leq 1\right\}
$$

Every Orlicz sequence space contains a subspace that is isomorphic to $\ell^{p}$, for some $1 \leq p<\infty$ or $c_{0}$ ([15], Theorem 4.a.9).

In the recent past lot of work has been done on sequence spaces defined by Orlicz functions by Altin et al. [16], Et et al. ([17, 18]), Tripathy et al. ([19-21]), and Mohiuddine et al. ([22-25]).

Given an Orlicz function $\varphi$, the Orlicz-Cesáro mean sequence spaces is defined by

$$
\begin{aligned}
& \operatorname{ces}_{\varphi}=\left\{u=\left(u_{i}\right) \in \omega: \rho(\beta u)<\infty \text { for some } \beta>0\right\}, \\
& \rho(u)=\sum_{i=0}^{\infty} \phi\left(\frac{\sum_{j=0}^{i}\left|u_{j}\right|}{i+1}\right) .
\end{aligned}
$$

$\left(\operatorname{ces}_{\varphi},\|\|.\right)$ is a Banach space with the Luxemburg norm given by

$$
\|u\|_{\text {ces }_{\varphi}}=\inf \left\{\beta>0: \rho\left(\beta^{-1} u\right) \leq 1\right\} .
$$

It seems that Orlicz-Cesáro mean sequence spaces $\operatorname{ces}_{\varphi}$ appeared for the first time in 1988, when Lim and Yee found their dual spaces [26]. Recently Cui, Hudzik, Petrot, Suantai, and Szymaszkiewicz obtained important properties of spaces $\operatorname{ces}_{\varphi}$ [27]. In 2007 Maligranda, Petrot, and Suantai showed that $\operatorname{ces}_{\varphi}$ is not B-convex, if $\varphi \in \Delta_{2}$ and $c e s_{\varphi} \neq 0$ [28]. The extreme points and strong $X$-points of $\operatorname{ces}_{\varphi}$ have been characterized by Foralewski, Hudzik, and Szymaszkiewicz in [29]. In the case when $\varphi(u)=u^{p}, 1 \leq p<\infty$, the space $c e s_{\varphi}$ is just a Cesáro sequence space $c e s_{p}$, with the norm given by

$$
\|u\|_{\text {ces }_{p}}=\left[\sum_{i=0}^{\infty}\left(\frac{\sum_{j=0}^{i}\left|u_{j}\right|}{i+1}\right)^{p}\right]^{1 / p} .
$$

It is well known that $\operatorname{ces}_{1}=\{0\}[30]$.

Definition 19 (see [31]). The Matuszewska Orlicz lower index $\alpha_{\varphi}$ of an Orlicz function $\varphi$ is defined as follows:

$$
\alpha_{\varphi}=\sup \left\{p>0: \exists_{K>0} \forall_{0<\lambda, t \leq 1} \varphi(\lambda t) \leq K t^{p} \varphi(\lambda)\right\} .
$$

Theorem 20 (see [31]). For any Orlicz function $\varphi$, we have $\alpha_{\varphi}>1$ if and only if $\ell_{\varphi} \subset$ ces $_{\varphi}$. In particular, if $\alpha_{\varphi}>1$ then $\operatorname{ces}_{\varphi} \neq\{0\}$.

Theorem 21 (see [31]). Let $\varphi_{1}$ and $\varphi_{2}$ be Orlicz functions. If there exist $b, t_{0}>0$ such that $\varphi_{2}\left(t_{0}\right)>0$ and $\varphi_{2}(t) \leq \varphi_{1}(b t)$ for all $t \in\left[0, t_{0}\right]$, then $\operatorname{ces}_{\varphi_{1}} \subset \operatorname{ces}_{\varphi_{2}}$.

Theorem 22 (see [31]). Let $\varphi_{1}$ and $\varphi_{2}$ be Orlicz functions and $\alpha_{\varphi_{1}}>1$, then $\operatorname{ces}_{\varphi_{1}} \subset \operatorname{ces}_{\varphi_{2}}$ if and only if there exist $b, t_{0}>0$ such that $\varphi_{2}\left(t_{0}\right)>0$ and $\varphi_{2}(t) \leq \varphi_{1}(b t)$ for all $t \in\left[0, t_{0}\right]$.

Definition 23 (see [2]). A class of linear sequence spaces $\mathbb{E}$ is called a special space of sequences (sss) having three properties:

(1) $e_{i} \in \mathbb{E}$ for all $i \in \mathbb{N}$,

(2) if $x=\left(x_{i}\right) \in w, y=\left(y_{i}\right) \in \mathbb{E}$ and $\left|x_{i}\right| \leq\left|y_{i}\right|$ for every $i \in \mathbb{N}$, then $x \in \mathbb{E}$, "i.e., $\mathbb{E}$ is solid",

(3) if $\left(x_{i}\right)_{i=0}^{\infty} \in \mathbb{E}$, then $\left(x_{[i / 2]}\right)_{i=0}^{\infty} \in \mathbb{E}$, wherever [i/2] means the integral part of $i / 2$.

Definition 24 (see [2]). A subclass of the special space of sequences is called a premodular (sss) if there is a function $\varrho: \mathbb{E} \longrightarrow[0, \infty[$ fulfilling the accompanying conditions:

(i) $\varrho(x) \geq 0$ for each $x \in \mathbb{E}$ and $\varrho(x)=0 \Longleftrightarrow x=\theta$, where $\theta$ is the zero element of $\mathbb{E}$,

(ii) there exists $L \geq 1$ such that $\varrho(\lambda x) \leq L|\lambda| \varrho(x)$ for all $x \in \mathbb{E}$, and for any scalar $\lambda$,

(iii) for some $K \geq 1$, we have $\varrho(x+y) \leq K(\varrho(x)+\varrho(y))$ for every $x, y \in \mathbb{E}$, 
(iv) if $\left|x_{i}\right| \leq\left|y_{i}\right|$ for all $i \in \mathbb{N}$, then $\varrho\left(\left(x_{i}\right)\right) \leq \varrho\left(\left(y_{i}\right)\right)$,

(v) for some $K_{0} \geq 1$, we have

$$
\varrho\left(\left(x_{i}\right)\right) \leq \varrho\left(\left(x_{[i / 2]}\right)\right) \leq K_{0} \varrho\left(\left(x_{i}\right)\right),
$$

(vi) the set of all finite sequences is $\varrho$-dense in $\mathbb{E}$. This means for each $x=\left(x_{i}\right)_{i=o}^{\infty} \in \mathbb{E}$ and for each $\varepsilon>0$ there exists $m \in \mathbb{N}$ such that $\varrho\left(\left(x_{i}\right)_{i=m}^{\infty}\right)<\varepsilon$,

(vii) there exists a constant $\xi>0$ such that $\varrho(\lambda, 0,0,0, \ldots) \geq \xi|\lambda| \varrho(1,0,0,0, \ldots)$ for any $\lambda \in \mathbb{R}$.

We denote $\left(\mathbb{E}_{\varrho}, \varrho\right)$ for the linear space $\mathbb{E}$ equipped with the metrizable topology generated by $\varrho$.

Theorem 25 (see [32]). If $X, Y$ are infinite dimensional Banach spaces and $\lambda_{i}$ is a monotonic decreasing sequence to zero, then there exists a bounded linear operator $T$ such that

$$
\frac{1}{16} \lambda_{3 i} \leq \alpha_{i}(T) \leq 8 \lambda_{i+1}
$$

Notations 26 (see [3]).

$S_{\mathbb{E}} \quad:=\quad\left\{S_{\mathbb{E}}(X, Y) ; X\right.$ and $Y$ are Banach Spaces $\}$, where

$$
\begin{aligned}
& S_{\mathbb{E}}(X, Y):=\left\{T \in \mathfrak{L}(X, Y):\left(\left(s_{i}(T)\right)_{i=0}^{\infty} \in \mathbb{E}\right\} .\right. \text { Also } \\
& S_{\mathbb{E}}^{a p p}:=\left\{S_{\mathbb{E}}^{a p p}(X, Y) ; X \text { and } Y \text { are Banach Spaces }\right\} \\
& \text { where } \\
& S_{\mathbb{E}}^{a p p}(X, Y):=\left\{T \in \mathfrak{L}(X, Y):\left(\left(\alpha_{i}(T)\right)_{i=0}^{\infty} \in \mathbb{E}\right\} .\right.
\end{aligned}
$$

Theorem 27 (see [3]). If $\mathbb{E}$ is a (sss), then $S_{\mathbb{E}}$ is an operator ideal.

The concept of pre-quasi operator ideal which is more general than the usual classes of operator ideal.

Definition 28 (see [3]). A function $g: \Omega \rightarrow[0, \infty)$ is said to be a pre-quasi norm on the ideal $\Omega$ fulfilling the accompanying conditions:

(1) for all $T \in \Omega(X, Y), g(T) \geq 0$ and $g(T)=0$ if and only if $T=0$,

(2) there exists a constant $L \geq 1$ such that $g(\beta T) \leq$ $L|\beta| g(T)$, for all $T \in \Omega(X, Y)$ and $\beta \in \mathbb{R}$,

(3) there exists a constant $K \geq 1$ such that $g\left(T_{1}+T_{2}\right) \leq$ $K\left[g\left(T_{1}\right)+g\left(T_{2}\right)\right]$, for all $T_{1}, T_{2} \in \Omega(X, Y)$,

(4) there exists a constant $C \geq 1$ such that if $P \in$ $\mathfrak{L}\left(X_{0}, X\right), R \in \Omega(X, Y)$, and $T \in \mathfrak{L}\left(Y, Y_{0}\right)$, then $g(T R P) \leq C\|T\| g(R)\|P\|$, where $X_{0}$ and $Y_{0}$ are normed spaces.

Theorem 29 (see [3]). Every quasi norm on the ideal $\Omega$ is a pre-quasi norm on the ideal $\Omega$.

Here and after, we define $e_{i}=\{0,0, \ldots, 1,0,0, \ldots\}$ where 1 appears at the $i^{\text {th }}$ place for all $i \in \mathbb{N}$.

\section{Main Results}

We give here the conditions on Orlicz-Cesáro mean sequence spaces $c e s_{\varphi}$ such that the class $S_{c e s_{\varphi}}$ of all bounded linear operators between arbitrary Banach spaces with its sequence of $s$-numbers which belong to $c e s_{\varphi}$ forms an operator ideal.

Theorem 30. If $\varphi$ is an Orlicz function satisfying $\Delta_{2}$-condition and $\alpha_{\varphi}>1$, then $S_{\text {ces }_{\varphi}}$ is an operator ideal.

Proof. (1-i) Let $x, y \in \operatorname{ces}_{\varphi}$. Since $\varphi$ is nondecreasing, convex, and satisfying $\Delta_{2}$-condition, we get for some $k>0$ that

$$
\begin{aligned}
& \sum_{n=0}^{\infty} \varphi\left(\frac{\sum_{i=0}^{n}\left|x_{i}+y_{i}\right|}{n+1}\right) \\
& \quad \leq k\left[\sum_{n=0}^{\infty} \varphi\left(\frac{\sum_{i=0}^{n}\left|x_{i}\right|}{n+1}\right)+\sum_{n=0}^{\infty} \varphi\left(\frac{\sum_{i=0}^{n}\left|y_{i}\right|}{n+1}\right)\right]<\infty,
\end{aligned}
$$

then $x+y \in \operatorname{ces}_{\varphi}$.

(1-ii) Let $\lambda \in \mathbb{R}$ and $x \in$ ces $_{\varphi}$, and since $\varphi$ is convex and satisfying $\Delta_{2}$-condition, we get for some $k>0$ that

$$
\sum_{n=0}^{\infty} \varphi\left(\frac{\sum_{i=0}^{n}\left|\lambda x_{i}\right|}{n+1}\right) \leq|\lambda| k \sum_{n=0}^{\infty} \varphi\left(\frac{\sum_{i=0}^{n}\left|x_{i}\right|}{n+1}\right)<\infty,
$$

then $\lambda x \in \operatorname{ces}_{\varphi}$; from (1-i) and (1-ii) $\operatorname{ces}_{\varphi}$ is a linear space. Since $e_{n} \in \ell_{\varphi}$, for all $n \in \mathbb{N}$ and $\alpha_{\varphi}>1$, then from Theorem 20, we get $e_{n} \in \operatorname{ces}_{\varphi}$, for all $n \in \mathbb{N}$.

(2) Let $\left|x_{n}\right| \leq\left|y_{n}\right|$ for all $n \in \mathbb{N}$ and $y \in$ ces $_{\varphi}$; since $\varphi$ is nondecreasing, then we have

$$
\sum_{n=0}^{\infty} \varphi\left(\frac{\sum_{i=0}^{n}\left|x_{i}\right|}{n+1}\right) \leq \sum_{n=0}^{\infty} \varphi\left(\frac{\sum_{i=0}^{n}\left|y_{i}\right|}{n+1}\right)<\infty,
$$

and we get $x \in \operatorname{ces}_{\varphi}$.

(3) Let $\left(x_{n}\right) \in \operatorname{ces}_{\varphi}$. Since $\varphi$ is satisfying $\Delta_{2}$-condition, we get for some $k>0$ that

$$
\sum_{n=0}^{\infty} \varphi\left(\frac{\sum_{i=0}^{n}\left|x_{[i / 2]}\right|}{n+1}\right) \leq(k+1) \sum_{n=0}^{\infty} \varphi\left(\frac{\sum_{i=0}^{n}\left|x_{i}\right|}{n+1}\right)<\infty,
$$

then $\left(x_{[n / 2]}\right) \in \operatorname{ces}_{\varphi}$. Then ces $_{\varphi}$ is a (sss); hence by Theorem 27, $S_{\text {ces }_{\varphi}}$ is an operator ideal.

Corollary 31. $S_{\text {ces }_{q}}$ is an operator ideal, if $1<q<\infty$.

We give the conditions on Orlicz-Cesáro mean sequence spaces $\operatorname{ces}_{\varphi}$ such that the ideal of the finite rank operators is dense in $S_{\text {ces }_{\varphi}}(X, Y)$.

Theorem 32. $S_{\text {ces }_{\varphi}}(X, Y)=\overline{F(X, Y)}$, if $\varphi$ is an Orlicz function satisfying $\Delta_{2}$-condition and $\alpha_{\varphi}>1$.

Proof. Let us define $\varrho(u)=\sum_{i=0}^{\infty} \varphi\left(\sum_{j=0}^{i}\left|u_{j}\right| /(i+1)\right)$ on $\operatorname{ces}_{\varphi}$. First, we have to show that $\overline{F(X, Y)} \subseteq S_{c e s_{\varphi}}(X, Y)$. Since $\alpha_{\varphi}>1$, we have $e_{i} \in \operatorname{ces}_{\varphi}$ for each $i \in \mathbb{N}$ and $\varphi$ is an 
Orlicz function satisfying $\Delta_{2}$-condition, so for each finite operator $P \in F(X, Y)$, i.e., we obtain $\left(s_{i}(P)\right)_{i=0}^{\infty}$ which contains only finitely many terms different from zero; hence $P \in$ $S_{c e s_{\varphi}}(X, Y)$. Currently we prove that $S_{c e s_{\varphi}}(X, Y) \subseteq \overline{F(X, Y)}$; let $P \in S_{c e s_{\varphi}}(X, Y)$; we have $\left(s_{i}(P)\right)_{i=0}^{\infty} \in$ ces $_{\varphi}$; and hence $\varrho\left(s_{i}(P)\right)_{i=0}^{\infty}<\infty$. By taking $\varepsilon \in(0,1)$, hence there exists a $i_{0} \in \mathbb{N}-\{0\}$ such that $\varrho\left(\left(s_{i}(P)\right)_{i=i_{0}}^{\infty}\right)<\varepsilon / 9 \delta C^{2}$ for some $c \geq 1$, where $\delta=\max \left\{1, \sum_{i=i_{0}}^{\infty} \varphi(1 /(i+1))\right\}$. As $s_{i}(P)$ is decreasing for every $i \in \mathbb{N}$ and $\varphi$ is nondecreasing, we have

$$
\begin{aligned}
i_{0} \varphi\left(s_{2 i_{0}}(P)\right) & \leq \sum_{i=i_{0}+1}^{2 i_{0}} \varphi\left(\frac{\sum_{j=0}^{i} s_{j}(P)}{i+1}\right) \\
& \leq \sum_{i=i_{0}}^{\infty} \varphi\left(\frac{\sum_{j=0}^{i} s_{j}(P)}{i+1}\right)<\frac{\varepsilon}{9 \delta C^{2}} .
\end{aligned}
$$

Hence, there exists $B \in F_{2 i_{0}}(X, Y)$ such that rank $B \leq 2 i_{0}$ and

$$
i_{0} \varphi(\|P-B\|) \leq \sum_{i=i_{0}+1}^{2 i_{0}} \varphi\left(\frac{\sum_{j=0}^{i}\|P-B\|}{i+1}\right)<\frac{\varepsilon}{9 \delta C^{2}} .
$$

Since $\varphi$ is right continuous at 0 and nondecreasing, then on considering this

$$
\|P-B\|<\frac{\varepsilon}{6 C^{2} i_{0} \delta} .
$$

Let $k_{1}>0, k_{2}>0$ and $C=\max \left\{1, k_{1}, k_{2}\right\}$, since $\varphi$ is Orlicz function and by using (22), (23), and (24), we have

$$
\begin{aligned}
& d(P, B)=\varrho\left(s_{i}(P-B)\right)_{i=0}^{\infty}=\sum_{i=0}^{3 i_{0}-1} \varphi \\
& \cdot\left(\frac{\sum_{j=0}^{i} s_{j}(P-B)}{i+1}\right)+\sum_{i=3 i_{0}}^{\infty} \varphi\left(\frac{\sum_{j=0}^{i} s_{j}(P-B)}{i+1}\right) \\
& \leq \sum_{i=0}^{3 i_{0}-1} \varphi\left(\frac{\sum_{j=0}^{i}\|P-B\|}{i+1}\right)+\sum_{i=i_{0}}^{\infty} \varphi \\
& \cdot\left(\frac{\sum_{j=0}^{i+2 i_{0}} s_{j}(P-B)}{i+1}\right) \leq 3 i_{0} \varphi(\|P-B\|)+\sum_{i=i_{0}}^{\infty} \varphi \\
& \cdot\left(\frac{\sum_{j=0}^{i+2 i_{0}} s_{j}(P-B)}{i+1}\right) \leq 3 i_{0} \varphi(\|P-B\|) \\
& +\sum_{i=i_{0}}^{\infty} \varphi\left(\frac{\sum_{j=0}^{2 i_{0}-1} s_{j}(P-B)+\sum_{j=2 i_{0}}^{i+2 i_{0}} s_{j}(P-B)}{i+1}\right) \\
& \leq 3 i_{0} \varphi(\|P-B\|)+k_{1}\left[\sum_{i=i_{0}}^{\infty} \varphi\left(\frac{\sum_{j=0}^{2 i_{0}-1} s_{j}(P-B)}{i+1}\right)\right.
\end{aligned}
$$

$$
\begin{aligned}
& \left.+\sum_{i=i_{0}}^{\infty} \varphi\left(\frac{\sum_{j=2 i_{0}}^{i+2 i_{0}} s_{j}(P-B)}{i+1}\right)\right] \leq 3 i_{0} \varphi(\|P-B\|) \\
& +k_{1}\left[\sum_{i=i_{0}}^{\infty} \varphi\left(\frac{\sum_{j=0}^{2 i_{0}-1}\|P-B\|}{i+1}\right)\right. \\
& \left.+\sum_{i=i_{0}}^{\infty} \varphi\left(\frac{\sum_{j=0}^{i} s_{j+2 i_{0}}(P-B)}{i+1}\right)\right] \leq 3 i_{0} \varphi(\|P-B\|) \\
& +2 i_{0} k_{1} k_{2}\|P-B\| \sum_{i=i_{0}}^{\infty} \varphi\left(\frac{1}{i+1}\right)+k_{1} \sum_{i=i_{0}}^{\infty} \varphi \\
& \cdot\left(\frac{\sum_{j=0}^{i} s_{j}(P)}{i+1}\right) \leq 3 i_{0} \varphi(\|P-B\|)+2 i_{0} C^{2} \| P \\
& -B \| \sum_{i=i_{0}}^{\infty} \varphi\left(\frac{1}{i+1}\right)+C \sum_{i=i_{0}}^{\infty} \varphi\left(\frac{\sum_{j=0}^{i} s_{j}(P)}{i+1}\right)<\varepsilon .
\end{aligned}
$$

Corollary 33. $S_{\text {ces }_{p}}(X, Y)=\overline{F(X, Y)}$, if $1<p<\infty$.

We express the accompanying theorem without verification; these can be set up utilizing standard procedure.

Theorem 34. The function $g(P)=\sum_{i=0}^{\infty} \varphi\left(\sum_{j=0}^{i}\left|s_{j}(P)\right| /(i+1)\right)$ is a pre-quasi norm on $S_{c e s_{\varphi}}$, if $\varphi$ is an Orlicz function satisfying $\Delta_{2}$-condition and $\alpha_{\varphi}>1$.

We give the sufficient conditions on Orlicz-Cesáro mean sequence spaces $\operatorname{ces}_{\varphi}$ such that the components of the prequasi operator ideal $S_{c e s_{\varphi}}$ are complete.

Theorem 35. If $X$ and $Y$ are Banach spaces, $\varphi$ is an Orlicz function satisfying $\Delta_{2}$-condition and $\alpha_{\varphi}>1$, then $\left(S_{c e s_{\varphi}}(X, Y), g\right)$ is a pre-quasi Banach operator ideal.

Proof. Since $\varphi$ is an Orlicz function satisfying $\Delta_{2^{-}}$ condition, then the function $g(P)=\varrho\left(\left(s_{n}(P)\right)_{n=0}^{\infty}\right)=$ $\sum_{n=0}^{\infty} \varphi\left(\sum_{m=0}^{n}\left|s_{m}(P)\right| /(n+1)\right)$ is a pre-quasi norm on $S_{c e s_{\varphi}}$. Let $\left(P_{m}\right)$ be a Cauchy sequence in $S_{c s_{\varphi}}(X, Y)$. Since $\mathfrak{L}(X, Y) \supseteq S_{c e s_{\varphi}}(X, Y)$ and $\alpha_{\varphi}>1$, we can find a constant $\xi>0$ such that

$$
\begin{aligned}
g\left(P_{i}-P_{j}\right) & =\varrho\left(\left(s_{n}\left(P_{i}-P_{j}\right)\right)_{n=0}^{\infty}\right) \\
& \geq \varrho\left(s_{0}\left(P_{i}-P_{j}\right), 0,0,0, \ldots\right) \\
& =\varrho\left(\left\|P_{i}-P_{j}\right\|, 0,0,0, \ldots\right) \\
& \geq \xi\left\|P_{i}-P_{j}\right\| \varrho(1,0,0,0, \ldots),
\end{aligned}
$$

then $\left(P_{m}\right)_{m \in \mathbb{N}}$ is also a Cauchy sequence in $\mathfrak{L}(X, Y)$. While the space $\mathfrak{R}(X, Y)$ is a Banach space, there exists $P \in \mathfrak{Q}(X, Y)$ 
such that $\lim _{m \rightarrow \infty}\left\|P_{m}-P\right\|=0$, while $\left(s_{n}\left(P_{m}\right)\right)_{n=0}^{\infty} \in \operatorname{ces}_{\varphi}$ for every $m \in \mathbb{N}$. Since $\varrho$ is continuous at $\theta$ and for some $K \geq 1$, we obtain

$$
\begin{aligned}
g(P)= & \varrho\left(\left(s_{n}(P)\right)_{n=0}^{\infty}\right)=\varrho\left(\left(s_{n}\left(P-P_{m}+P_{m}\right)\right)_{n=0}^{\infty}\right) \\
\leq & K \varrho\left(\left(s_{[n / 2]}\left(P-P_{m}\right)\right)_{n=0}^{\infty}\right) \\
& +K \varrho\left(\left(\alpha_{[n / 2]}\left(P_{m}\right)_{n=0}^{\infty}\right)\right) \\
\leq & K \varrho\left(\left(\left\|P_{m}-P\right\|\right)_{n=0}^{\infty}\right)+K \varrho\left(\left(s_{n}\left(P_{m}\right)_{n=0}^{\infty}\right)\right) \\
< & \infty
\end{aligned}
$$

we have $\left(s_{n}(P)\right)_{n=0}^{\infty} \in c e s_{\varphi}$, and then $P \in S_{c e s_{\varphi}}(X, Y)$.

Corollary 36. If $X$ and $Y$ are Banach spaces and $1<q<$ $\infty$, then $\left(S_{\text {ces }_{q}}(X, Y), g\right)$ is quasi Banach operator ideal, where $g(P)=\varrho\left(\left(s_{n}(P)\right)_{n=0}^{\infty}\right)=\left[\sum_{n=0}^{\infty}\left(\sum_{m=0}^{n}\left|s_{m}(P)\right| /(n+1)\right)^{q}\right]^{1 / q}$.

Theorem 37. Let $\varphi_{1}, \varphi_{2}$ be Orlicz functions and $\alpha_{\varphi_{1}}>1$. For any infinite dimensional Banach spaces $X, Y$ and if there exist $b, t_{0}>0$ such that $\varphi_{2}\left(t_{0}\right)>0$ and $\varphi_{2}(t) \leq \varphi_{1}(b t)$ for all $t \epsilon$ $\left[0, t_{0}\right]$, it is true that

$$
S_{\operatorname{ces}_{\varphi_{1}}}^{a p p}(X, Y) \varsubsetneqq S_{\operatorname{ces}_{\varphi_{2}}}^{a p p}(X, Y) \varsubsetneqq \mathfrak{L}(X, Y) .
$$

Proof. Let $X$ and $Y$ be infinite dimensional Banach spaces and there exist $b, t_{0}>0$ such that $\varphi_{2}\left(t_{0}\right)>0$ and $\varphi_{2}(t) \leq \varphi_{1}(b t)$ for all $t \in\left[0, t_{0}\right]$; if $P \in S_{\text {ces }_{\varphi_{1}}}^{a p p}(X, Y)$, then $\left(\alpha_{n}(P)\right) \in$ ces $_{\varphi_{1}}$. From Theorems 21, 22, and 25, we have $\operatorname{ces}_{\varphi_{1}} \subset \operatorname{ces}_{\varphi_{2}}$; hence $P \in S_{c e s_{\varphi_{2}}}^{a p p}(X, Y)$. It is easy to see that $S_{c e s_{\varphi_{2}}}^{a p p}(X, Y) \subset \mathfrak{L}(X, Y)$.

Corollary 38. For any infinite dimensional Banach spaces $X$, $Y$, and $1<p<q<\infty$, then $S_{c e s}^{a p p}(X, Y) \varsubsetneqq S_{\text {ces }_{q}}^{a p p}(X, Y) \varsubsetneqq$ $\mathfrak{Q}(X, Y)$.

We now study some properties of the pre-quasi Banach operator ideal $S_{\text {ces }_{\varphi}}$.

Theorem 39. The pre-quasi Banach operator ideal $\left(S_{c e s_{\varphi}}, g\right)$ is injective, if the s-number sequence is injective.

Proof. Let $T \in \mathfrak{L}(X, Y)$ and $P \in \mathfrak{Q}\left(Y, Y_{0}\right)$ be any metric injection. Assume that $P T \in S_{\text {ces }_{\varphi}}\left(X, Y_{0}\right)$, then $\varrho\left(s_{n}(P T)\right)<\infty$. Since the $s$-number sequence is injective, we have $s_{n}(P T)=$ $s_{n}(T)$, for all $T \in \mathfrak{Q}(X, Y), n \in \mathbb{N}$. So $\varrho\left(s_{n}(T)\right)=\varrho\left(s_{n}(P T)\right)<$ $\infty$. Hence $T \in S_{\text {ces }_{\varphi}}(X, Y)$ and clearly $g(T)=g(P T)$ is verified.

Remark 40. The pre-quasi Banach operator ideal $\left(S_{c e s_{\varphi}}^{\text {Weyl }}, g\right)$ and the pre-quasi Banach operator ideal $\left(S_{c e s_{\varphi}}^{G e l}, g\right)$ are injective pre-quasi Banach operator ideal.

Theorem 41. The pre-quasi Banach operator ideal $\left(S_{c e s_{\varphi}}, g\right)$ is surjective, if the s-number sequence is surjective.
Proof. Let $T \in \mathfrak{L}(X, Y)$ and $P \in \mathfrak{Q}\left(X_{0}, X\right)$ be any metric surjection. Suppose that $T P \in S_{c e s_{\varphi}}\left(X_{0}, Y\right)$, then $\varrho\left(s_{n}(T P)\right)<$ $\infty$. Since the $s$-number sequence is surjective, we have $s_{n}(T P)=s_{n}(T)$, for all $T \in \mathfrak{L}(X, Y), n \in \mathbb{N}$. So $\varrho\left(s_{n}(T)\right)=$ $\varrho\left(s_{n}(T P)\right)<\infty$. Hence $T \in S_{\text {ces }_{\varphi}}(X, Y)$ and clearly $g(T)=$ $g(T P)$ is verified.

Remark 42. The pre-quasi Banach operator ideal $\left(S_{c e s_{\varphi}}^{\text {Chang }}\right.$, $g$ ) and the pre-quasi Banach operator ideal $\left(S_{c e s_{\varphi}}^{K o l}, g\right)$ are surjective pre-quasi Banach operator ideal.

Likewise, we have the accompanying inclusion relations between the pre-quasi Banach operator ideals.

Theorem 43. (1) $S_{c e s_{\varphi}}^{a p p} \subseteq S_{c e s_{\varphi}}^{K o l} \subseteq S_{c e s_{\varphi}}^{\text {Chang }} \subseteq S_{c e s_{\varphi}}^{H i l b}$.

(2) $S_{\text {ces }_{\varphi}}^{a p p} \subseteq S_{\text {ces }_{\varphi}}^{\text {Gel }} \subseteq S_{\operatorname{ces}_{\varphi}}^{\text {Weyl }} \subseteq S_{\text {ces }_{\varphi}}^{H i l b}$.

Proof. Since $h_{n}(T) \leq y_{n}(T) \leq d_{n}(T) \leq \alpha_{n}(T)$ and $h_{n}(T) \leq$ $x_{n}(T) \leq c_{n}(T) \leq \alpha_{n}(T)$ for every $n \in \mathbb{N}$ and $\varrho$ is nondecreasing, we obtain

$$
\begin{aligned}
& \varrho\left(h_{n}(T)\right) \leq \varrho\left(y_{n}(T)\right) \leq \varrho\left(d_{n}(T)\right) \leq \varrho\left(\alpha_{n}(T)\right), \\
& \varrho\left(h_{n}(T)\right) \leq \varrho\left(x_{n}(T)\right) \leq \varrho\left(c_{n}(T)\right) \leq \varrho\left(\alpha_{n}(T)\right) .
\end{aligned}
$$

Hence the result is as follows.

We presently express the dual of the pre-quasi operator ideal formed by different $s$ - number sequences.

Theorem 44. The pre-quasi operator ideal $S_{\text {ces }}^{\text {Hilb }}$ is completely symmetric and the pre-quasi operator ideal $S_{c e s_{\varphi}}^{a p p}$ is symmetric.

Proof. Since $h_{n}\left(T^{\prime}\right)=h_{n}(T)$ and $\alpha_{n}\left(T^{\prime}\right) \leq \alpha_{n}(T)$, for all $T \in$ $\mathfrak{L}(X, Y)$, we have $S_{c e s_{\varphi}}^{H i l b}=\left(S_{c e s_{\varphi}}^{H i l b}\right)^{\prime}$ and $S_{c e s_{\varphi}}^{a p p} \subseteq\left(S_{c e s_{\varphi}}^{a p p}\right)^{\prime}$.

In perspective on Theorem 13, we express the following result without proof.

Theorem 45. The pre-quasi operator ideal $S_{c e s_{\varphi}}^{\mathrm{Kol}} \subseteq\left(S_{c e s_{\varphi}}^{\mathrm{Gel}}\right)^{\prime}$ and $S_{c e s_{\varphi}}^{G e l}=\left(S_{\text {ces }_{\varphi}}^{\text {Kol }}\right)^{\prime}$. In addition if $T$ is a compact operator from $X$ to $Y$, then $S_{c e s_{\varphi}}^{K o l}=\left(S_{\text {ces }_{\varphi}}^{G e l}\right)^{\prime}$.

In perspective on Theorem 14, we express the following result without proof.

Theorem 46. The pre-quasi operator ideal $S_{c e s_{\varphi}}^{\text {Chang }}=\left(S_{c e s_{\varphi}}^{\text {Weyl }}\right)^{\prime}$ and $S_{\text {ces }}^{\text {Weyl }}=\left(S_{\text {ces }_{\varphi}}^{\text {Chang }}\right)^{\prime}$.

Theorem 47. If $\varphi$ is an Orlicz function satisfying $\Delta_{2}$-condition and $\alpha_{\varphi}>1$, then the pre-quasi Banach operator ideal $S_{\text {ces }_{\varphi}}^{a p p}$ is small.

Proof. Since $\varphi$ is an Orlicz function and $\alpha_{\varphi}>1$, take $\beta=\sum_{i=0}^{\infty} \varphi(1 /(i+1))$. Then $\left(S_{c e s_{\varphi}}^{a p p}, g\right)$, where $g(T)=$ 
$\varrho\left(\left(\alpha_{n}(T)\right)_{n=0}^{\infty}\right)=(1 / \beta) \sum_{n=0}^{\infty} \varphi\left(\sum_{m=0}^{n} \alpha_{m}(T) /(n+1)\right)$ is a prequasi Banach operator ideal. Let $X$ and $Y$ be any two Banach spaces. Assume that $S_{c e s_{\varphi}}^{a p p}(X, Y)=\mathfrak{Q}(X, Y)$, then there exists a constant $C>0$ such that $g(T) \leq C\|T\|$ for all $T \in \mathfrak{L}(X, Y)$. Suppose that $X$ and $Y$ are infinite dimensional Banach spaces. Then by Dvoretzky's theorem [8] for $m \in \mathbb{N}$, we have quotient spaces $X / M_{m}$ and subspaces $N_{m}$ of $Y$ which can be mapped onto $\ell_{2}^{m}$ by isomorphisms $V_{m}$ and $B_{m}$ such that $\left\|V_{m}\right\|\left\|V_{m}^{-1}\right\| \leq$ 2 and $\left\|B_{m}\right\|\left\|B_{m}^{-1}\right\| \leq 2$. Consider $I_{m}$ be the identity map on $\ell_{2}^{m}, P_{m}$ be the quotient map from $X$ onto $X / M_{m}$, and $Q_{m}$ be the natural embedding map from $N_{m}$ into $Y$. Let $v_{n}$ be the Bernstein numbers [7], then

$$
\begin{aligned}
1 & =v_{n}\left(I_{m}\right)=v_{n}\left(B_{m} B_{m}^{-1} I_{m} V_{m} V_{m}^{-1}\right) \\
& \leq\left\|B_{m}\right\| v_{n}\left(B_{m}^{-1} I_{m} V_{m}\right)\left\|V_{m}^{-1}\right\| \\
& =\left\|B_{m}\right\| v_{n}\left(Q_{m} B_{m}^{-1} I_{m} V_{m}\right)\left\|V_{m}^{-1}\right\| \\
& \leq\left\|B_{m}\right\| d_{n}\left(Q_{m} B_{m}^{-1} I_{m} V_{m}\right)\left\|V_{m}^{-1}\right\| \\
& =\left\|B_{m}\right\| d_{n}\left(Q_{m} B_{m}^{-1} I_{m} V_{m} Q_{m}\right)\left\|V_{m}^{-1}\right\| \\
& \leq\left\|B_{m}\right\| \alpha_{n}\left(Q_{m} B_{m}^{-1} I_{m} V_{m} Q_{m}\right)\left\|V_{m}^{-1}\right\|,
\end{aligned}
$$

for $1 \leq i \leq m$. Now since $\varphi$ is nondecreasing and having $\Delta_{2^{-}}$ condition, we have

$$
\begin{aligned}
& \sum_{j=0}^{i}(1) \leq \sum_{j=0}^{i}\left\|B_{m}\right\| \alpha_{j}\left(Q_{m} B_{m}^{-1} I_{m} V_{m} P_{m}\right)\left\|V_{m}^{-1}\right\| \Longrightarrow \\
& \frac{1}{i+1}(i+1) \leq\left\|B_{m}\right\|\left(\frac{1}{i+1} \sum_{j=0}^{i} \alpha_{j}\left(Q_{m} B_{m}^{-1} I_{m} V_{m} P_{m}\right)\right) \\
& \cdot\left\|V_{m}^{-1}\right\| \Longrightarrow \\
& \varphi(1) \leq L\left(\left\|B_{m}\right\|\left\|V_{m}^{-1}\right\|\right) \\
& \cdot \varphi\left(\frac{1}{i+1} \sum_{j=0}^{i} \alpha_{j}\left(Q_{m} B_{m}^{-1} I_{m} V_{m} P_{m}\right)\right)
\end{aligned}
$$

Therefore

$$
\begin{gathered}
\sum_{i=0}^{m} \varphi(1) \leq L\left\|B_{m}\right\|\left\|V_{m}^{-1}\right\| \sum_{i=0}^{m} \varphi \\
\cdot\left(\frac{1}{i+1} \sum_{j=0}^{i} \alpha_{j}\left(Q_{m} B_{m}^{-1} I_{m} V_{m} P_{m}\right)\right) \Longrightarrow \\
\frac{\varphi(1)}{\beta}(m+1) \leq L\left\|B_{m}\right\|\left\|V_{m}^{-1}\right\| \frac{1}{\beta} \sum_{i=0}^{m} \varphi \\
\cdot\left(\frac{1}{i+1} \sum_{j=0}^{i} \alpha_{j}\left(Q_{m} B_{m}^{-1} I_{m} V_{m} P_{m}\right)\right) \Longrightarrow
\end{gathered}
$$

$$
\begin{aligned}
& \frac{\varphi(1)}{\beta}(m+1) \leq L\left\|B_{m}\right\|\left\|V_{m}^{-1}\right\| g\left(Q_{m} B_{m}^{-1} I_{m} V_{m} P_{m}\right) \Longrightarrow \\
& \frac{\varphi(1)}{\beta}(m+1) \leq L C\left\|B_{m}\right\|\left\|V_{m}^{-1}\right\|\left\|Q_{m} B_{m}^{-1} I_{m} V_{m} P_{m}\right\| \Longrightarrow \\
& \frac{\varphi(1)}{\beta}(m+1) \leq L C\left\|B_{m}\right\|\left\|V_{m}^{-1}\right\|\left\|Q_{m} B_{m}^{-1}\right\|\left\|I_{m}\right\|\left\|V_{m} P_{m}\right\| \\
& \quad=L C\left\|B_{m}\right\|\left\|V_{m}^{-1}\right\|\left\|B_{m}^{-1}\right\|\left\|I_{m}\right\|\left\|V_{m}\right\| \Longrightarrow \\
& \frac{\varphi(1)}{\beta}(m+1) \leq 4 L C,
\end{aligned}
$$

for some $L \geq 1$. Thus we arrive at a contradiction since $m$ is arbitrary. Hence $X$ and $Y$ both cannot be infinite dimensional when $S_{\text {ces }_{\varphi}}^{a p p}(X, Y)=\mathfrak{Q}(X, Y)$.

Theorem 48. If $\varphi$ is an Orlicz function satisfying $\Delta_{2}$-condition and $\alpha_{\varphi}>1$, then the pre-quasi Banach operator ideal $S_{c e s_{\varphi}}^{K o l}$ is small.

Corollary 49. If $p \in(1, \infty)$, then the quasi Banach operator ideal $S_{\text {ces }}^{a p p}$ is small.

Corollary 50. If $p \in(1, \infty)$, then the quasi Banach operator ideal $S_{\text {ces }}^{K o l}$ is small.

\section{Examples}

We give some examples which support our main results.

Example 1. Let $\varphi$ be an Orlicz function; the subspace $c e s_{\varphi}^{h}$ of all order continuous elements of $c e s_{\varphi}$ is defined as [27]

$$
\begin{aligned}
& \operatorname{ces}_{\varphi}^{h} \\
& \quad=\left\{x \in \operatorname{ces}_{\varphi}: \forall_{k>0} \exists_{n_{k} \in \mathbb{N}} \sum_{n=n_{k}}^{\infty} \varphi\left(\frac{k}{n} \sum_{i=1}^{n}\left|x_{i}\right|\right)<\infty\right\} .
\end{aligned}
$$

If $\varphi$ is an Orlicz function satisfying $\Delta_{2}$-condition and $\alpha_{\varphi}>1$, then the following conditions are satisfied:

(1) $S_{c e s}^{h}$ is an operator ideal.

(2) $S_{c e s_{\varphi}^{h}}(X, Y)=\overline{F(X, Y)}$.

(3) If $X$ and $Y$ are Banach spaces, then $\left(S_{c e s}^{h}(X, Y), g\right)$ is pre-quasi Banach operator ideal.

(4) The pre-quasi Banach operator ideal $S_{c e s_{\varphi}^{h}}^{a p p}$ is small.

Proof. Since $\varphi$ is an Orlicz function satisfying $\Delta_{2}$-condition and $\alpha_{\varphi}>1$, then from Theorem (5) in [31] we have $\operatorname{ces}_{\varphi}^{h}=$ $\operatorname{ces}_{\varphi}$ which completes the proof. 
Example 2. Let $\varphi$ be defined as

$$
\varphi(t)=a_{l} t^{l}+a_{l-1} t^{l-1}+\ldots+a_{1} t,
$$

where $a_{i}>0$ for all $1 \leq i \leq l, l \in \mathbb{N}, l>1$ and $t \geq 0$.

It is clear that $\varphi$ is an Orlicz function and $\alpha_{\varphi}=l>1$. Also $\varphi$ is satisfying $\Delta_{2}$-condition since

$$
\limsup _{t \rightarrow 0^{+}} \frac{\varphi(2 t)}{\varphi(t)} \leq 2^{l}<\infty .
$$

Then the following conditions are satisfied:

(1) $S_{c e s_{\varphi}}$ is an operator ideal.

(2) $S_{c e s_{\varphi}}(X, Y)=\overline{F(X, Y)}$.

(3) If $X$ and $Y$ are Banach spaces, then $\left(S_{c e s_{\varphi}}(X, Y), g\right)$ is pre-quasi Banach operator ideal.

(4) The pre-quasi Banach operator ideal $S_{c e s_{\varphi}}^{a p p}$ is small.

In the following two examples we will explain the importance of the sufficient conditions.

Example 3. Let $\varphi$ be defined as

$$
\varphi(t)= \begin{cases}0 & \text { if } t=0, \\ \frac{-t}{\ln t} & \text { if } t \in\left(0, \frac{1}{e}\right], \\ \frac{3}{2} e t^{2}-t+\frac{1}{2 e} & \text { if } t \in\left(\frac{1}{e}, \infty\right) .\end{cases}
$$

It is clear that $\varphi$ is an Orlicz function. Since $\sum_{n=1}^{\infty} \varphi(1 / n)=$ $\sum_{n=1}^{\infty}(1 / n \ln n)=\infty$, hence $c e s_{\varphi}=\{0\}$. The space $S_{c e s_{\varphi}}$ is not operator ideal since $I_{K} \notin S_{c e s_{\varphi}}$. Also since $\varphi$ is convex function and for $p>1$, we have

$$
\begin{aligned}
\lim _{t \rightarrow 0^{+}} \frac{\varphi(\lambda t)}{\varphi(\lambda) t^{p}} & =\lim _{t \rightarrow 0^{+}} \frac{t^{1-p} \ln \lambda}{\ln \lambda t} \\
& =\lim _{t \rightarrow 0^{+}}(1-p) t^{1-p} \ln \lambda=\infty,
\end{aligned}
$$

for all $\lambda \in(0,1]$, then $\alpha_{\varphi}=1$. Although $\varphi$ is satisfying $\Delta_{2}-$ condition since

$$
\limsup _{t \rightarrow 0^{+}} \frac{\varphi(2 t)}{\varphi(t)}=\limsup _{t \rightarrow 0^{+}} \frac{2 \ln t}{\ln 2 t} \leq 2<\infty .
$$

Example 4. Let $\varphi(u)=\int_{0}^{u} f(t) d t$, where $f(t)$ is defined as

$$
\begin{aligned}
& f(t) \\
& \quad= \begin{cases}0 & \text { if } t=0, \\
\frac{1}{n !} & \text { if } t \in\left[\frac{1}{(n+1) !}, \frac{1}{n !}\right) \text { for } n=1,2,3 \ldots, \\
t & \text { if } t \in[1, \infty) .\end{cases}
\end{aligned}
$$

It is clear that $\varphi$ is an Orlicz function. Let $T \in S_{c e s_{\varphi}}$ with $s_{n}(T)=1 / n$ ! for all $n \in \mathbb{N}$. We have for $n>2$ that

$$
\begin{aligned}
\varphi\left(s_{n}(2 T)\right) & =\int_{0}^{2 / n !} f(t) d t>\int_{1 / n !}^{2 / n !} f(t) d t \\
& >\int_{1 / n !}^{1 /(n-1) !} f(t) d t>\frac{1}{n !(n-1) !}, \\
n \varphi\left(s_{n}(T)\right) & =n \int_{0}^{1 / n !} f(t) d t \\
& <n \sup _{0 \leq t \leq 1 / n !} f(t) \int_{0}^{1 / n !} 1 d t<\frac{1}{n !(n-1) !} .
\end{aligned}
$$

Hence $2 T \notin S_{c e s_{\varphi}}$, so the space $S_{c e s_{\varphi}}$ is not operator ideal and $\varphi \notin \Delta_{2}$. Also since $\varphi$ is convex function and for $p>1$, we have

$$
\lim _{t \rightarrow 0^{+}} \frac{\varphi(\lambda t)}{\varphi(\lambda) t^{p}}=\lim _{t \rightarrow 0^{+}} t^{-p}=\infty
$$

for all $\lambda \in(0,1]$, then $\alpha_{\varphi}=1$.

\section{Data Availability}

The data used to support the findings of this study are available from the corresponding author upon request.

\section{Disclosure}

The authors received no financial support for the research, authorship, and or publication of this article.

\section{Conflicts of Interest}

The authors declare that have no conflicts of interest.

\section{Authors' Contributions}

All authors contributed equally to the writing of this paper. All authors read and approved the final manuscript.

\section{References}

[1] A. Pietsch, Operator Ideals, vol. 20, North-Holland Publishing Company, Amsterdam, The Netherlands, 1980.

[2] N. F. Mohamed and A. A. Bakery, "Mappings of type Orlicz and generalized Cesáro sequence space," Journal of Inequalities and Applications, vol. 2013, article 186, 2013.

[3] N. Faried and A. A. Bakery, "Small operator ideals formed by s numbers on generalized Cesáro and Orlicz sequence spaces," Journal of Inequalities and Applications, vol. 2018, no. 1, article $357,2018$.

[4] D. Costarelli and G. Vinti, "A quantitative estimate for the sampling kantorovich series in terms of the modulus of continuity in orlicz spaces," Constructive Mathematical Analysis, vol. 2, no. 1, pp. 8-14, 2019. 
[5] F. Altomare, "Iterates of markov operators and constructive approximation of semigroups," Constructive Mathematical Analysis, vol. 2, no. 1, pp. 22-39, 2019.

[6] A. Pietsch, Eigenvalues and s-Numbers, Cambridge University Press, New York, NY, USA, 1986.

[7] A. Pietsch, "s-numbers of operators in banach spaces," Studia Mathematica, vol. 51, pp. 201-223, 1974.

[8] A. Pietsch, Operator Ideals, VEB Deutscher Verlag DerWissenschaften, Berlin, Germany, 1978.

[9] C. V. Hutton, "On the approximation numbers of an operator and its adjoint," Mathematische Annalen, vol. 210, pp. 277-280, 1974.

[10] N. J. Kalton, "Spaces of compact operators," Mathematische Annalen, vol. 208, pp. 267-278, 1974.

[11] Å. Lima and E. Oja, "Ideals of finite rank operators, intersection properties of balls, and the approximation property," Studia Mathematica, vol. 133, no. 2, pp. 175-186, 1999.

[12] M. A. Krasnoselskii and Y. B. Rutickii, Convex Functions and Orlicz Spaces, Gorningen, Netherlands, 1961.

[13] W. Orlicz and Ü. Raume, " $\mathrm{L}$," Bulletin International de l'Academie Polonaise des Sciences et des Lettres Série A, pp. 93$107,1936$.

[14] J. Lindenstrauss and L. Tzafriri, "On Orlicz sequence spaces," Israel Journal of Mathematics, vol. 10, pp. 379-390, 1971.

[15] J. Lindenstrauss and L. Tzafriri, Classical Banach Spaces, vol. 92 of I. Sequence spaces, Ergebnisse der Mathematik und ihrer Grenzgebiete, Springer-Verlag, Berlin, Germany, 1977.

[16] Y. Altin, M. Et, and B. C. Tripathy, "The sequence space $\left|\bar{N}_{p}\right|(M, r, q, s)$ on seminormed spaces," Applied Mathematics and Computation, vol. 154, no. 2, pp. 423-430, 2004.

[17] M. Et, L. P. Lee, and B. C. Tripathy, "Strongly almost $(V, \lambda)\left(\Delta^{r}\right)-$ summable sequences defined by Orlicz functions," Hokkaido Mathematical Journal, vol. 35, no. 1, pp. 197-213, 2006.

[18] M. Et, Y. Altin, B. Choudhary, and B. C. Tripathy, "On some classes of sequences defined by sequences of Orlicz functions," Mathematical Inequalities \& Applications, vol. 9, no. 2, pp. 335342, 2006.

[19] B. C. Tripathy and S. Mahanta, "On a class of difference sequences related to the $l^{p}$ space defined by Orlicz functions," Mathematica Slovaca, vol. 57, no. 2, pp. 171-178, 2007.

[20] B. C. Tripathy and H. Dutta, "On some new paranormed difference sequence spaces defined by Orlicz functions," Kyungpook Mathematical Journal, vol. 50, no. 1, pp. 59-69, 2010.

[21] B. C. Tripathy and B. Hazarika, "I-convergent sequences spaces defined by Orlicz function," Acta Mathematicae Applicatae Sinica, vol. 27, no. 1, pp. 149-154, 2011.

[22] S. A. Mohiuddine and B. Hazarika, "Some classes of ideal convergent sequences and generalized difference matrix operator," Filomat, vol. 31, no. 6, pp. 1827-1834, 2017.

[23] S. A. Mohiuddine and K. Raj, "Vector valued Orlicz-Lorentz sequence spaces and their operator ideals," Journal of Nonlinear Sciences and Applications. JNSA, vol. 10, no. 2, pp. 338-353, 2017.

[24] S. Abdul Mohiuddine, K. Raj, and A. Alotaibi, "Generalized spaces of double sequences for Orlicz functions and boundedregular matrices over $n$-normed spaces," Journal of Inequalities and Applications, vol. 2014, article 332, 2014.

[25] S. A. Mohiuddine, S. K. Sharma, and D. A. Abuzaid, "Some seminormed difference sequence spaces over n-normed spaces defined by a musielak-orlicz function of order $(\alpha, \beta)$," Journal of Function Spaces, vol. 2018, Article ID 4312817, 11 pages, 2018.
[26] S.-K. Lim and P. Y. Lee, "An Orlicz extension of Cesáro sequence spaces," Roczniki Polskiego Towarzystwa Matematycznego. Seria I. Commentationes Mathematicae. Prace Matematyczne, vol. 28, no. 1, pp. 117-128, 1988.

[27] Y. Cui, H. Hudzik, N. Petrot, S. Suantai, and A. Szymaszkiewicz, "Basic topological and geometric properties of Cesáro-Orlicz spaces," The Proceedings of the Indian Academy of Sciences Mathematical Sciences, vol. 115, no. 4, pp. 461-476, 2005.

[28] L. Maligranda, N. Petrot, and S. Suantai, "On the James constant and B-convexity of Cesáro and Cesàro-Orlicz sequence spaces," Journal of Mathematical Analysis and Applications, vol. 326, pp. 312-326, 2007.

[29] P. Foralewski, H. Hudzik, and A. Szymaszkiewicz, "Local rotundity structure of Cesáro-Orlicz sequence spaces," Journal of Mathematical Analysis and Applications, vol. 345, no. 1, pp. 410-419, 2008.

[30] G. M. Leibowitz, "A note on the Cesáro sequence spaces," Tamkang Journal of Mathematics, vol. 2, no. 2, pp. 151-157, 1971.

[31] D. Kubiak, "A note on Cesáro-Orlicz sequence spaces," Journal of Mathematical Analysis and Applications, vol. 349, no. 1, pp. 291-296, 2009.

[32] B. M. Makarov and N. Faried, "Some properties of operator ideals constructed by s numbers (in Russian)," in Theory of Operators in Functional Spaces, pp. 206-211, Academy of Science, Siberian section, Novosibirsk, Russia, 1977. 


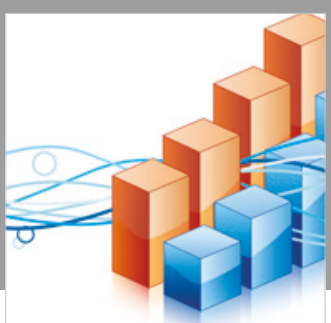

Advances in

Operations Research

\section{-n-m}
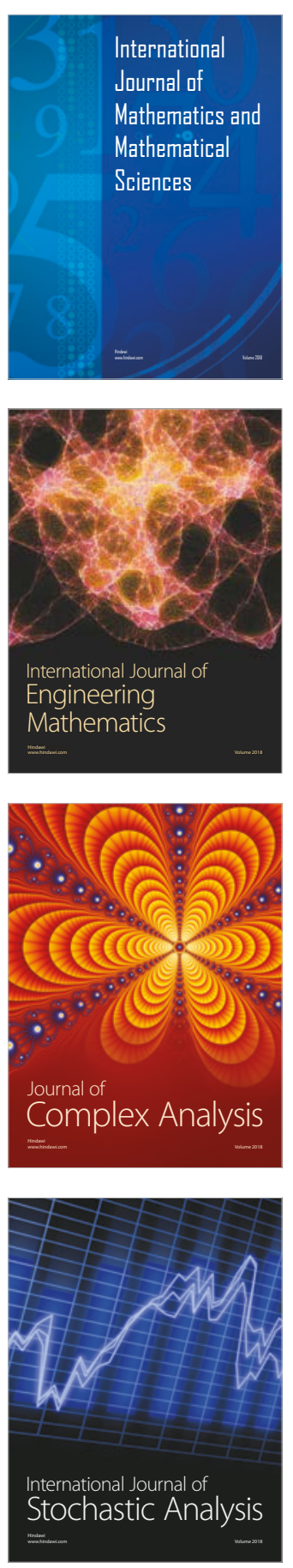
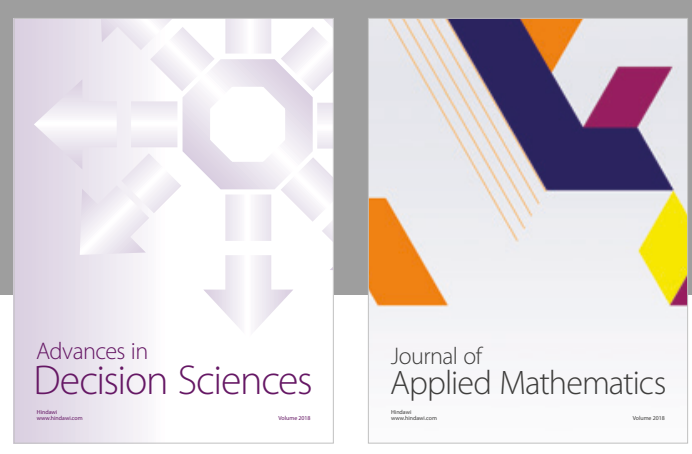

Journal of

Applied Mathematics
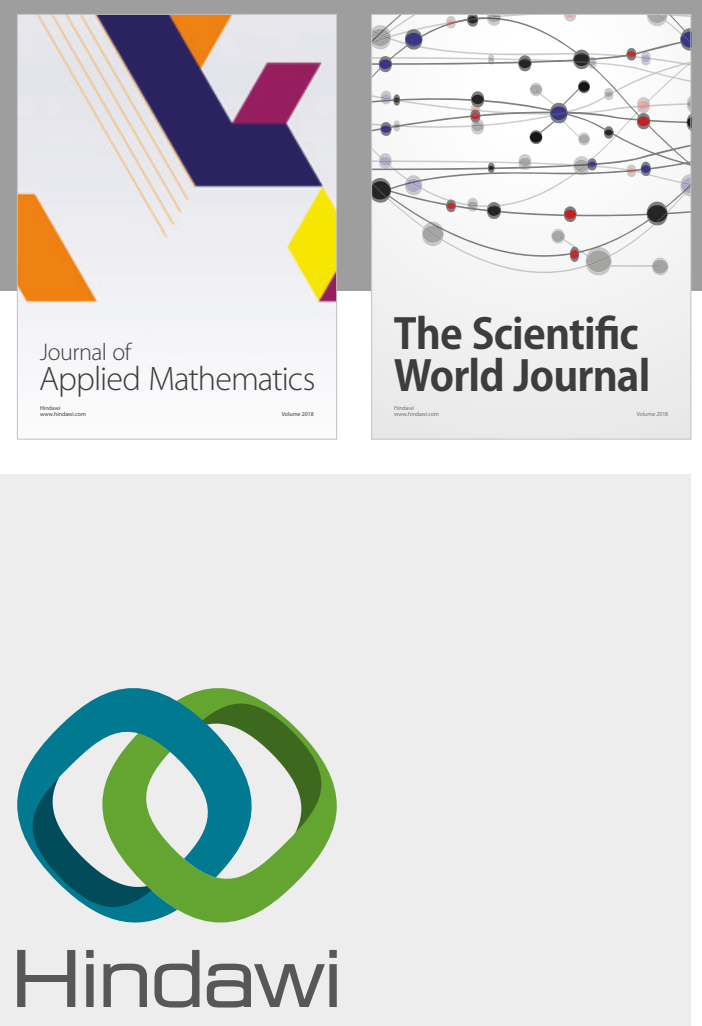

Submit your manuscripts at

www.hindawi.com

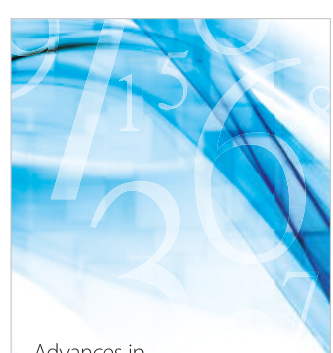

Advances in
Numerical Analysis
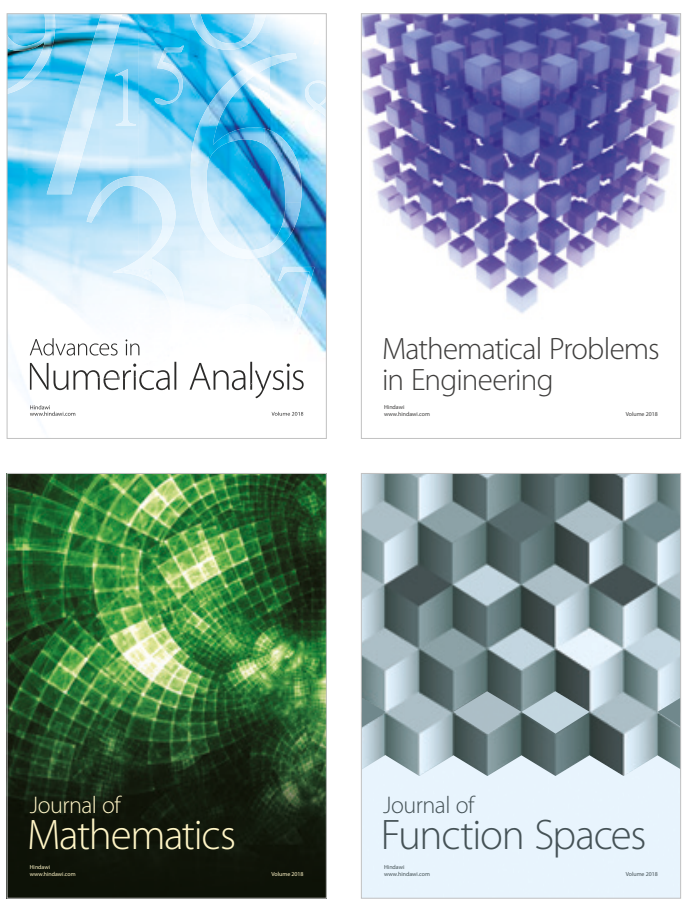

Mathematical Problems in Engineering

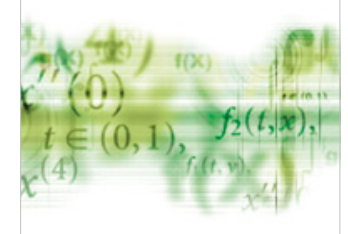

International Journal of

Differential Equations

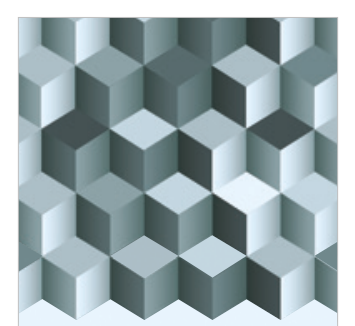

Journal of

Function Spaces

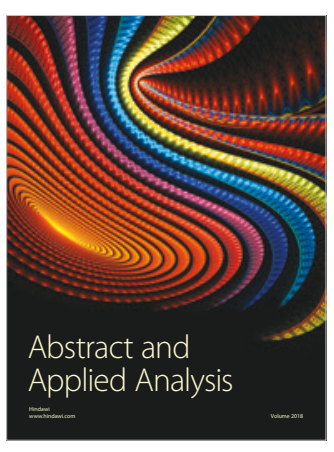

The Scientific

World Journal

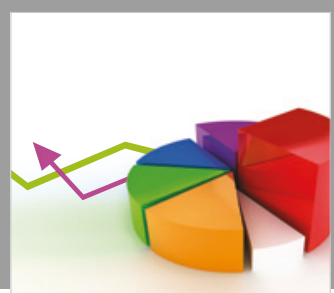

Journal of

Probability and Statistics
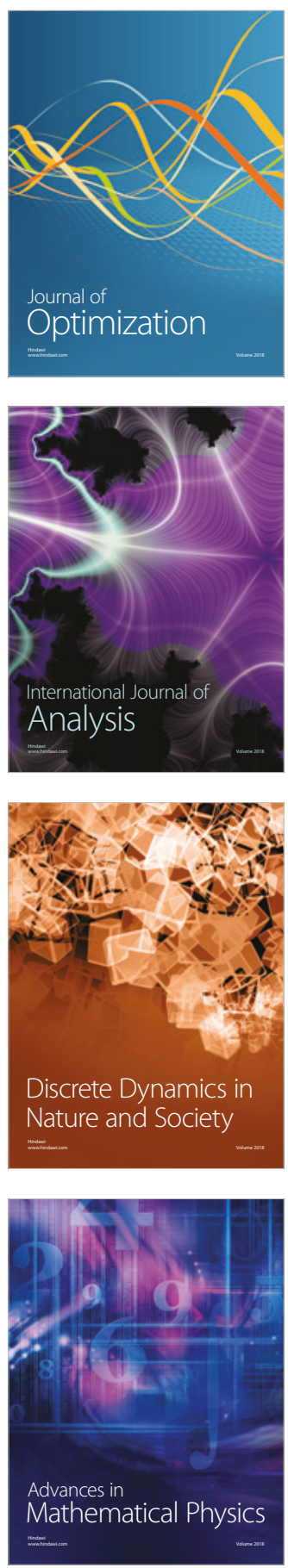